\title{
A Influência das Condições Ambientais na Emergência Hospitalar pelo Estado Físico e Emocional do Consumidor
}

\author{
The Influence of Environmental Conditions on Hospital Emergency by the \\ Consumer's Physical and Emotional State
}

\begin{abstract}
Alexandre de Oliveira Siqueira ${ }^{1 *}$, Taciana de Barros Jerônimo ${ }^{1}$, Marcus Augusto Vasconcelos Araújo ${ }^{2}$, Jamille Queiroz Leite ${ }^{1}$

${ }^{1}$ Universidade Federal de Pernambuco (UFPE), Pernambuco, Brasil.

${ }^{2}$ Centro Universitário UniFBV/Wyden.
\end{abstract}

\section{INFO ARTIGO}

\section{Palavras-chave:}

Ambiente de serviços

hospitalar;

Psicologia ambiental;

Percepção do ambiente;

Emergência hospitalar.

\begin{abstract}
RES U MO
A presente pesquisa teve como objetivo analisar a relação entre as variáveis ambientais presentes na sala de espera de uma emergência hospitalar privada, os estados físico e emocional do consumidor e os seus reflexos na percepção e na resposta emocional no uso do serviço. Para tanto, foi analisado um hospital de grande porte em Recife a partir da condução de uma survey com 300 consumidores entre pacientes e acompanhantes. Os dados foram analisados por meio de regressão logística. Os resultados demonstraram que as condições físicas do ambiente que mais se relacionaram com a percepção ambiental do consumidor foram cor, aroma, layout e temperatura e que os estados físico e emocional impactam negativamente na percepção e na resposta emocional do consumidor, trazendo seis contribuições teóricas relevantes. A originalidade deste estudo se baseia no fato de não existir estudos empíricos que associem os estados físicos e emocionais dos consumidores com as condições ambientes, percepção e resposta emocional ao ambiente de serviços de emergência.
\end{abstract}

A B S T R A C T
This research aimed to analyze the relationship between the environmental
variables present in the waiting room of a private hospital emergency, the
physical and emotional states of the consumer and their reflexes on perception
and emotional response in the use of the service. Therefore, a large hospital
in Recife was analyzed by conducting a survey with 300 consumers between
patients and caregivers. Data were analyzed by logistic regression. The results
showed that the physical conditions of the environment that most related to
the consumer's environmental perception were color, aroma, layout and
temperature and that the physical and emotional states negatively impact
the consumer's perception and emotional response, bringing six relevant
theoretical contributions. The originality of this study is based on the fact that
there are no empirical studies that associate the physical and emotional states
of consumers with the environmental conditions, perception and emotional
response to the emergency services environment; comparative analysis
between patients and caregivers in emergency service settings.

\begin{tabular}{l}
\hline A R T I C L E I N F O \\
\hline Keywords: \\
Hospital services \\
environment; \\
Environmental psychology; \\
Perception of the \\
environment; \\
Hospital emergency.
\end{tabular}

* Correspondência para autor:

alex.pisciano@gmail.com (Siqueira,A.O.) (ORCID: 0000-0001-6765-2247), taciana.barros@gmail.com

(Jerônimo, T.B.) (ORCID: 0000-0003-1643-3030), marcusaugusto77@hotmail.com (Araújo, M. A.V.)

(ORCID: 0000-0003-4559-0860), jamillequeiroz@gmail.com (Leite, J.Q.)(ORCID: 0000-0001-5446-5178). 


\section{Introdução}

Em ambientes de serviços, as condições ambientais que precisam ser gerenciadas incluem não apenas os elementos físicos, mas também os sensoriais como temperatura, iluminação, ruído, música, odor e layout (BITNER, 1992). Neste sentido, diversos estudos tem reforçado que o planejamento do ambiente de serviços representa um elemento importante no processo de escolha e decisão de compra do consumidor uma vez que as condições ambientais detectadas pelos sentidos humanos podem afetar a interação dos consumidores com o serviço (BITNER, 1992, BAKER; PARASURAMAN, 1994; BABIN; ATTAWAY, 2000, VAN et al. 2016; SAVELLI; CIOPPI; TOMBARI; 2017; BUJISIC et al., 2017).

Especificamente no que concerne aos ambientes de serviços de saúde, os ambientes de espera em emergência representem a mais crítica das etapas da experiência de consumo, pois a espera nestes normalmente é caracterizada por situações de crise, em que os principais consumidores em cena (pacientes e acompanhantes) apresentam um estado emocional especialmente marcado pela ansiedade, insegurança, perda da independência, perda do poder de decisão, perda da identidade, do reconhecimento social e da autoestima (NOGUEIRA, 2003, BEUNINGEN, 2004; LEE, 2011; SHUV-AMI; SHALOM, 2017). Neles, a influência dos fatores ambienteis tende a ser ainda mais crítica, tendo em vista a que a percepção do tempo de espera para o atendimento que envolvem ansiedade e/ou desconforto físico, características normalmente presentes quando se busca um serviço de saúde, são mais desagradáveis (MCCOLL-KENNEDY et al., 2017), tornando o planejamento do ambiente de espera fundamental para se reduzir a ansiedade e melhorar a avaliação da qualidade do serviço hospitalar por parte dos pacientes (TAYLOR, 1994; ZAREI, 2015; PAI; CHARY, 2016).

Neste contexto, o objetivo do presente artigo é analisar a influência das condições ambientais na emergência hospitalar no estado físico e emocional dos consumidores. Para tanto, foi realizado um estudo que avaliou a relação entre as condições ambientais presentes na sala de espera de emergência de um grande hospital de Recife, bem como o estado físico e emocional e os reflexos destes na percepção e nas respostas emocionais dos pacientes-consumidores que por este ambiente passaram.

\section{O ambiente de serviços em gestão hospitalar}

Segundo Lyu et al. (2017), as características dos ambientes físicos em serviços têm o potencial não apenas de influenciar o comportamento dos consumidores, mas, sobretudo, de criar posicionamentos competitivos para organizações. Nesse sentido, diversas pesquisas têm avaliado os ambientes físicos de estabelecimentos de saúde com o objetivo de examinar o impacto de ambientes físicos nas experiências dos consumidores (KOTLER et al., 2000; BECKER, SWEENEY, PARSONS; 2008; HOLDER; BERNDT, 2011; SHENG; SIGUAW; SIMPSON, 2016). Nesta linha, Hui e Tse (1996), Lee (2011) e Sahoo e Mitra (2016) demonstram que a maioria dos consumidores chegam as instalações de saúde apresentado sentimentos como dor, angústia, preocupação e ansiedade, cuja intensidade tende a influenciar a avaliação global do serviço.

Uma das ferramentas que podem ser utilizadas para a avaliação de serviços é a atmosfera de loja, que engloba o ambiente em que o serviço é prestado e possui uma implicação direta na qualidade do espaço delimitador do ambiente a partir das suas quatro dimensões: visual (cor, brilho, tamanho, formas), auricular (som), olfativa (cheiro, frescor) e tátil (suavidade, temperatura). A partir do momento em que se planeja e manipula estes fatores ambientais, pode-se, então, criar um efeito positivo na avaliação dos clientes em relação ao serviço que está sendo consumido (KOTLER, 1973). Para hospitais, em específico, a atmosfera tem grande relevância, pois o consumidor geralmente se desloca até as instalações físicas do prestador de serviço para utilizá-lo (SAHOO; MITRA, 2016).

Nesta linha, trabalhos recentes tem avaliado o tema da atmosfera de serviço em hospitais, com foco nos ambientes de internação, a exemplo dos apartamentos e enfermarias e gerenciamento dos seus elementos sensoriais, como cores, iluminação, ruídos, som ambiente, aromas, temperatura e layout espacial (NARANG et al., 2015; SAHOO; GHOSH, 2016).

Em se tratando dos efeitos das cores em ambientes de serviços, verifica-se que o gerenciamento dessas variáveis tem como objetivo atrair fisicamente, de forma visual, os consumidores, bem como contribuir para qualidade percebida pelo usuário (TRIANTAFILLIDOU; SIOMKOS; PAPAFILIPPAKI, 2017). 
Já com relação ao ruído em ambientes de serviços, Bailey e Timmons (2005) e Ugolini, Rossato e Baccarani (2014) afirmam que a sua percepção é subjetiva e influenciada por vários fatores como cultura, aspectos sociais e sensibilidade auditiva individual. Schuster e Weber (2003) descrevem que o nível de ruído em ambientes hospitalares pode aumentar a pressão sanguínea, a batida do coração e estimular a liberação de adrenalina dos pacientes. Adicionalmente, ruídos intermitentes podem provocar percepção ambiental negativa pelo consumidor, enquanto sons ambientais muito altos causam irritação (BAILEY; TIMMONS, 2005; WOLZAK, 2012).

Em seu estudo sobre som ambiente em hospitais, Tansik e Routhieaux (1999) apontam que o uso da música estava relacionado à diminuição do estresse e ao aumento do relaxamento em comparação com os momentos em que nenhuma música foi utilizada em um ambiente de espera. Evans (2002), Zhu e Meyers-levy (2005), Soars (2009) e Peter e Olson, (2009) complementam que sons ambientes em baixa frequência causam percepção ambiental positiva pelo consumidor.

Quanto à temperatura, estudos apontam que a agressão e a probabilidade de tumulto crescem em ambientes com altas temperaturas (SANDERS; BRIZZOLARA, 1982; HOWARTH; HOFFMAN, 1984; ANDERSON, 1989; WU; WANG, 2015). Quando o cliente está se sentindo confortável, o estado afetivo tende a melhorar. Os autores ainda destacam que há temperaturas que as pessoas consideram mais agradáveis no ambiente de serviço. Dessa forma, temperaturas fora de uma classificação de conforto pelo consumidor, a exemplo de temperaturas baixas, são prováveis provocadores de um estado emocional negativo ao consumidor (DONOVAN; ROSSITER, 1982; SANDERS; BRIZZOLARA, 1982; HOWARTH; HOFFMAN, 1984; ANDERSON, 1989; BITNER, 1992; BAKER; CAMERON, 1996).

A atmosfera de loja aborda o uso das evidências físicas. Elas são utilizadas para criar ambientes de serviço e sua influência sobre as percepções e os comportamentos dos indivíduos é denominado de psicologia ambiental (HOFFMAN, BATESON; 2003). Para ajudar a explicar os efeitos do ambiente de serviço sobre o comportamento do consumidor, a psicologia ambiental trabalha com o modelo estímuloorganismo-resposta de Mehrabian e Russel (1974), citado por Donovan e Rossiter (1982), buscando compreender a influência da evidência física no comportamento dos consumidores. Este artigo analisa a percepção das variáveis ambientais de acordo com o modelo de estímulo de Mehrabian e Russel (1974).

As percepções desses ambientes se fundamentam em Donovan e Rossiter (1982), como percepções ambientais positivas e negativas. As repostas das pessoas ao ambiente podem ser caracterizadas, conforme os autores, como comportamentos de prazer ou agitação/afastamento. Nesse sentido, estudos apontam que uma percepção ambiental positiva pelo consumidor tende a provocar respostas emocionais de prazer, assim como uma percepção ambiental negativa pelo consumidor produz respostas emocionais de agitação (DONOVAN; ROSSITER, 1982; BITNER, 1992; SOLOMON, 2008; ZOMERDIJK; VOSS, 2010).

\section{Hipóteses de pesquisa e modelo teórico}

Com base no referencial teórico, são apresentadas as 10 hipóteses testadas na presente pesquisa no quadro 1 , onde são estão indicadas as relações que as compõem, considerando as variáveis independentes (dimensões ambientais), e as dependentes (percepção e respostas emocionais):

Quadro 1: Hipóteses de pesquisa.

\begin{tabular}{|c|l|l|}
\hline Hipótese & \multicolumn{1}{|c|}{ Descrição } & \multicolumn{1}{|c|}{ Autores } \\
\hline H1 & $\begin{array}{l}\text { A presença de som ambiental em baixa frequência } \\
\text { produz percepção ambiental positiva pelo } \\
\text { consumidor. }\end{array}$ & $\begin{array}{l}\text { EVANS, 2002; ZHU; MEYERS-LEVY, 2005; } \\
\text { SOARS, 2009; PETER; OLSON, 2009. }\end{array}$ \\
\hline H2 & $\begin{array}{l}\text { A presença de cores suaves ambientais produz } \\
\text { percepção ambiental positiva pelo consumidor. }\end{array}$ & $\begin{array}{l}\text { HOFFMAN; BATESON, 2003; SIDWELL, 2004; } \\
\text { DALKE et al, 2005; PARK; FARR, 2007; } \\
\text { SOLOMON, 2008; SOARS, 2009. }\end{array}$ \\
\hline H3 & $\begin{array}{l}\text { A presença de aromas ambientais agradáveis } \\
\text { produz percepção ambiental positiva pelo } \\
\text { consumidor. }\end{array}$ & $\begin{array}{l}\text { MORRIN; RATNESHWAR, 2000; HOFFMAN, } \\
\text { BATESON, 2003; MILOTIC, 2003; CHAPPELL, } \\
\text { 2004; CORBETT, 2006; SOLOMON, 2008; ZEMKE; } \\
\text { SHOEMAKER, 2008; COSTA, 2009. }\end{array}$ \\
\hline
\end{tabular}




\begin{tabular}{|c|c|c|}
\hline H4 & $\begin{array}{l}\text { O conforto do layout produz percepção ambiental } \\
\text { positiva pelo consumidor. }\end{array}$ & 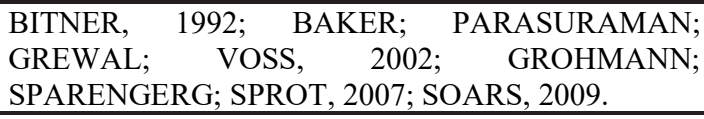 \\
\hline H5 & $\begin{array}{l}\text { A presença de ruídos intermitentes ambientais } \\
\text { produz percepção ambiental negativa pelo } \\
\text { consumidor. }\end{array}$ & $\begin{array}{l}\text { SCHUSTER; WEBER, 2003; BAILEY; TIMMONS, } \\
2005 .\end{array}$ \\
\hline H6 & $\begin{array}{l}\text { A presença de luzes incandescentes produz } \\
\text { percepção ambiental negativa pelo consumidor. }\end{array}$ & $\begin{array}{l}\text { BABIN, HARDESTY; SUTER, 2003; HOFFMAN; } \\
\text { BATESON, 2003; DALKE et al., 2005; PARK; } \\
\text { FARR, 2007; SOLOMON, 2008; QUARTIER; } \\
\text { CHRISTIAANS; VAN CLEEMPOEL, 2008; SOARS, } \\
2009 \text {. }\end{array}$ \\
\hline H7 & $\begin{array}{l}\text { A presença de temperaturas baixas ambientais } \\
\text { produz percepção ambiental negativa pelo } \\
\text { consumidor. }\end{array}$ & $\begin{array}{l}\text { DONOVAN; ROSSITER, 1982; } \text { SANDERS; } \\
\text { BRIZZOLARA, 1982; HOWARTH; HOFFMAN, } \\
\text { 1984; ANDERSON, 1989; BITNER, 1992; BAKER; } \\
\text { CAMERON, 1996. }\end{array}$ \\
\hline H8 & $\begin{array}{l}\text { Percepção ambiental positiva pelo consumidor } \\
\text { produz respostas emocionais positivas. }\end{array}$ & \multirow{2}{*}{$\begin{array}{lll}\text { KOTLER, 1973; DONOVAN; ROSSITER, } & 1982 ; \\
\text { BITNER, 1992; TURLEY; MILLIMAN, 2000; } & \text { ARNEILL; DEVLIN, 2002; GREWAL et al; 2003; } \\
\text { BEUNINGEN, 2004; KEILLOR; } \quad \text { HULT; } & \\
\text { KANDEMIR, 2004; DALKE et al, 2005; SOLOMON, } \\
\text { 2008; ZOMERDIJK; VOSS, 2010. }\end{array}$} \\
\hline H9 & $\begin{array}{l}\text { Percepção ambiental negativa pelo consumidor } \\
\text { produz respostas emocionais negativas. }\end{array}$ & \\
\hline H10a & $\begin{array}{l}\text { Os estados físico e emocional do consumidor } \\
\text { diminuem a percepção ambiental positiva e } \\
\text { resposta emocional positivas produzidas pelo } \\
\text { consumidor. }\end{array}$ & \multirow{2}{*}{$\begin{array}{l}\text { BEUNINGEN, 2004; GARCIA, 2005; GODOI, 2008; } \\
\text { BORBA, 2009; MINADEO, 2010; LEE, 2011, } \\
\text { SAHOO; MITRA, } 2016 .\end{array}$} \\
\hline $\mathrm{H} 10 \mathrm{~b}$ & $\begin{array}{l}\text { Os estados físico e emocional do consumidor } \\
\text { aumentam a percepção ambiental negativa e } \\
\text { resposta emocional negativas produzidas pelo } \\
\text { consumidor. }\end{array}$ & \\
\hline
\end{tabular}

Fonte: Desenvolvido pelos autores (2019).

A Figura 1 apresenta as 10 hipóteses propostas simultaneamente em um modelo interrelacional, desenvolvido com base no referencial teórico sobre servicescape (BITNER, 1992), Healthcare Servicescape (LEE, 2011; SAHOO; MITRA, 2016), atmosfera de loja (KOTLER, 1973) e no modelo estímuloorganismo-resposta de Mehrabian e Russell (1974), citado por Donovan e Rossiter (1982).

Figura 1: Modelo da influência do ambiente nas respostas emocionais do consumidor.

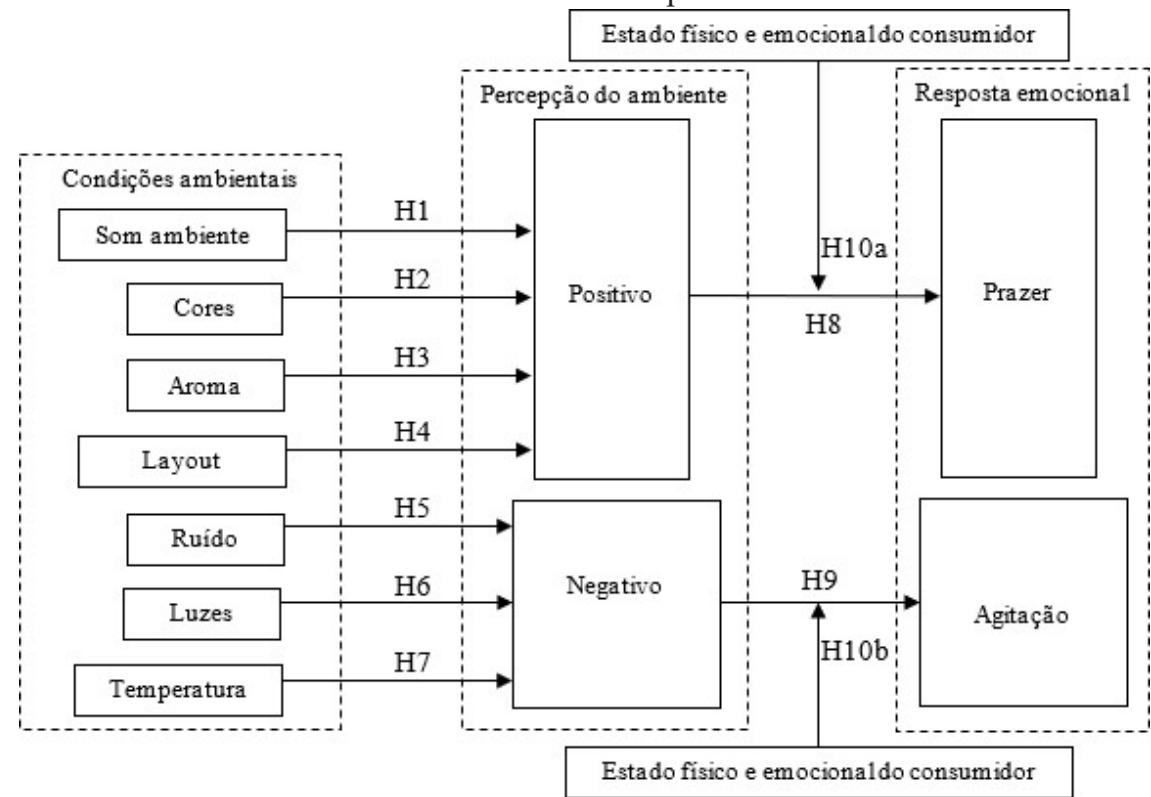

Fonte: Adaptado de Kotler (1973), Donovan e Rossiter (1982), Bitner (1992), Lee (2011) e Sahoo e Mitra

Fator (percepção ambiental): (2016).

- Espaço (instalações, equipamentos, áreas físicas), 
- Tempo (termo de atividade),

- Valor e atitude (regras e procedimentos internos). Fator (resposta emocional):

- Cognitivo (pensamento, crença, expectativa para a intensidade emocional),

- Psicológico (alterações resultantes do alerta emocional),

- Comportamental (sinais exteriores das emoções que estão sendo vivenciadas). Fator (condições ambientais):

- Som +, cor +, aroma +, layout +, ruído +, luzes +, temperatura. Fator (estado físico):

- $\sum$ dos três fatores anteriores.

\section{Metodologia}

Para a operacionalização da pesquisa, foi conduzido um estudo de caso no contexto de um hospital privado de grande porte do Recife. A escolha do hospital em Recife foi em função não só da conveniência, como também importância desse mercado no segmento de hospitais (G1, 2014; CREMEPE, 2014). Tratou-se de uma população com perfil da região nordeste e de uma capital, o que é importante para o entendimento de como se comporta o modelo desta pesquisa com este tipo de público. Optou-se por buscar indivíduos que procuram por serviços de emergência. Segundo estudo do IBPT (Instituto Brasileiro de Planejamento e Tributação) os serviços privados de saúde (hospitais, clínicas e laboratórios) vêm crescendo nos últimos anos. De 2011 a 2016, houve 47,04\% de aumento de faturamento (DIÁRIO DO GRANDE ABC, 2016), $12,8 \%$ de faturamento de planos de saúde em 2016 e crescimento de lucro de 70,6\% em 2016 comparado a 2015 (VALOR ECONÔMICO, 2017).

Segundo Yin (2005), estudos de caso podem ser conduzidos através de levantamentos e, em seguida, aplicadas técnicas estatísticas para análise dos dados, sendo esta pesquisa classificada como um estudo de caso de abordagem descritiva quantitativa, observacional e transversal, como eixo metodológico fundamental (GIL, 1999; SOUZA et al., 2002). Ou seja, a pesquisa foi conduzida a partir de uma amostra não-probabilística por conveniência (CHURCHILL, 1999).

No presente estudo, foi obtida uma amostra de 300 consumidores pesquisados, entre pacientes e acompanhantes. Foi realizado um levantamento (survey) por meio de questionário estruturado, aplicado aos consumidores da emergência, tendo sido obtido um percentual de $100 \%$ de questionários válidos, não havendo nenhum descarte já que todos os respondentes foram entrevistados diretamente pelos autores desta pesquisa.

Para a condução da pesquisa foi utilizado um instrumento de coleta de dados contendo 28 questões retratando os construtos analisados e outras quatro questões voltadas para a identificação de dados demográficos dos respondentes. Os questionários foram aplicados em pacientes e acompanhantes no setor de emergência do hospital estudado. No quadro 2 são apresentados os autores utilizados para elaboração do questionário. A escolha das escalas, por construto, baseou-se fundamentalmente nos instrumentos já desenvolvidos por esses autores.

Quadro 2: Escalas utilizadas no instrumento de coleta de dados.

\begin{tabular}{|c|c|c|}
\hline CONSTRUTOS & ESCALAS & AUTORES \\
\hline Som ambiente & \multirow{8}{*}{ Tipo Likert 5 pontos } & \multirow{8}{*}{$\begin{array}{l}\text { BITNER, 1992; LOURES, } \\
\text { 2003; BEUNINGEN, } 2004 .\end{array}$} \\
\hline Cores & & \\
\hline Aroma & & \\
\hline Layout & & \\
\hline Ruído & & \\
\hline Luzes & & \\
\hline Temperatura & & \\
\hline Percepção ambiental & & \\
\hline
\end{tabular}




\begin{tabular}{|c|c|c|}
\hline $\begin{array}{l}\text { Resposta emocional de } \\
\text { prazer (positiva) }\end{array}$ & \multirow{2}{*}{ Tipo Likert 5 pontos } & \multirow{2}{*}{$\begin{array}{l}\text { MEHRABIAN; RUSSELL, 1974; } \\
\text { SORIANO; FOXALL, 2001; } \\
\text { LOURES, 2003; ABREU; } \\
\text { TEIXEIRA, } 2007 .\end{array}$} \\
\hline $\begin{array}{l}\text { Resposta emocional de } \\
\text { agitação (negativa) }\end{array}$ & & \\
\hline $\begin{array}{l}\text { Estado físico e emocional do } \\
\text { consumidor }\end{array}$ & Intervalar & $\begin{array}{l}\text { LEE, 2011; SILVA; } \\
\text { DELIBERATO, 2009; } \\
\text { SOUSA et al., 2010. } \\
\end{array}$ \\
\hline
\end{tabular}

Fonte: Desenvolvido pelos autores (2019).

Os procedimentos de avaliação da inter-relação entre as questões de cada bloco foram realizados a partir da aplicação da técnica de Análise Fatorial Exploratória, objetivando identificar fatores latentes, bem como possíveis inaderências de questões individuais em cada dimensão identificada.

Um fator relevante que fundamenta a escolha da regressão logística é que este método não depende da suposição de normalidade das variáveis. Esta característica específica da regressão logística torna-a uma técnica mais robusta de maior aplicabilidade (HAIR et al., 2009). Tabachnick e Fidell (2001), apontam a regressão logística como sendo a melhor técnica multivariada quando a variável dependente não é paramétrica. Adicionalmente, quando associado à utilização de variáveis independentes binárias, este método permite a visualização clara do efeito que a presença de determinada variável independente gera na variável dependente, chamado de Odds Ratio ou razão de chances (TABACHNICK; FIDELL, 2001; HOSMER; LEMESHOW, 2010). Neste trabalho isso pode ser verificado que a variável dependente é o estado físico e emocional e as variáveis independentes são condições, percepções e estado emocional.

Para a utilização da Regressão Logística, foi utilizado o método Stepwise Backward LR, no qual o software executa um processo interativo em que, a partir do maior modelo possível com a presença de todas as variáveis independentes, são conduzidas várias etapas de redução até que sejam mantidas no modelo apenas as variáveis que se mostrem significantes para a relação de interesse (HAIR et al., 2009).

Neste sentido, objetivando melhorar o poder preditivo do modelo, além de gerar resultados assimiláveis na verificação das hipóteses, foi conduzida a transformação das variáveis ordinais em variáveis binárias. No que concerne o ponto de corte, a mediana foi a medida escolhida para transformação das escalas de 5 pontos. Foi arbitrado que respostas de valores inferiores a mediana, teriam seus valores transformados em 0 , tendo as demais (igual ou superior a mediana) transformadas em 1, seguindo as recomendações de Hair et al. (2009).

\section{Análise e discussão dos dados}

Nas análises estatísticas, foi utilizado o software SPSS v. 20. Especificamente em relação às técnicas utilizadas, conforme orientação de Hair et al. (2009), foram feitos testes de hipótese Kolmogorov-Smirnov e de Shapiro-Wilk, para a avaliação da normalidade dos dados. A partir dos testes realizados, nenhuma das dimensões analisadas apresentou uma forma de distribuição normal de dados. Também foi utilizado o coeficiente Alfa de Cronbach para verificar a confiabilidade e validade do instrumento de coleta de dados. Uma segunda etapa de análise, os resultados das questões foram avaliados individualmente, a partir da utilização de estatísticas descritivas e, por fim, na terceira etapa, foi calculado as relações entre as condições ambientais e percepção ambiental do consumidor a partir da utilização de regressão logística.

Para avaliar a confiabilidade ou consistência interna do instrumento de coleta, foi calculado o alfa de Cronbach. Hair et al. (2009) sugerem um alfa de Cronbach mínimo de 0,7 para que se garanta existir uma boa consistência na escala. O Alfa de Cronbach foi: 0,787 para condições ambientais; 0,824 para percepção ambiental, 0,724 para resposta emocional positiva, 0,636 para resposta emocional negativa e 0,747 para estado físico e emocional. Apesar da resposta emocional negativa ter apresentado um Alfa de Cronbach de 0,636, Malhotra (2006) considera como um item de confiabilidade moderada $(0,60 \leq \alpha \leq 0,70)$, sendo percebido que a exclusão de questões não levaria a melhora deste índice, demonstrando um caráter uniforme no entendimento dos entrevistados.

Foi conduzida também uma avaliação da existência de dimensões latentes em todos os blocos da pesquisa a partir de uma análise fatorial exploratória. Para avaliar a qualidade da análise, Hair et al. (2009) recomendam que seja avaliada a estatística de Kaiser-Meyer-Olkin (KMO) que indica a proporção da 
variância dos dados que pode ser considerada comum a todas as variáveis. Neste sentido, o resultado mais próximo de 1, mais adequada é a amostra à aplicação da análise fatorial.

O teste de esfericidade de Bartlett testa se a matriz de correlação é uma matriz identidade, o que indicaria que não há correlação entre os dados. Neste, verifica-se a existência de um nível de significância assumido em 5\% de modo a se rejeitar a hipótese nula. Para a análise do critério de significância da carga fatorial de cada variável nos fatores, Hair et al. (2009) recomendam o critério para considerar as cargas fatoriais como significativas: o mínimo aceitável para uma carga fatorial é 0,3 e, a partir de 0,500 de carga fatorial, as variáveis são consideradas significativas.

Observou-se que o KMO mínimo obtido foi de 0,647 para o bloco das condições ambientais e valores superiores a este para os blocos das demais dimensões, tornando o teste de esfericidade de Bartlett significativo $(\mathrm{p}<0,001)$. Na sequência, os resultados das cargas fatoriais de todas as variáveis superaram o valor de 0,5, com valor mínimo de 0,632, sendo consideradas, portanto, significativas (HAIR et al., 2009).

Em relação a análise descritiva da amostra foi observado que a frequência de consumo na instituição hospitalar pesquisada, $29 \%$ dos respondentes estavam acessando pela primeira vez ao hospital, enquanto uma parcela significativa de $54,7 \%$ já era considerada cliente, ou seja, estando pela terceira vez ou mais. No que concerne ao tempo aguardado na sala de espera pelo atendimento médico, $49 \%$ dos respondentes estavam aguardando a até apenas 20 minutos, intervalo esse que corrobora com o indicador estipulado de 30 minutos máximo para aguardar por atendimento em salas de espera de emergência (MELHORES PRÁTICAS, 2015).

Quanto à distribuição por gênero, 39,7\% dos respondentes foram homens e 60,3\% mulheres. Já com relação à faixa etária, a maioria dos respondentes tem um perfil adulto, com 86,7\% tendo entre 19 e 59 anos. O nível de escolaridade da amostra mostrou-se alto, com 53\% dos respondentes declarando possuir graduação completa e pós-graduação. Finalmente, no que concerne a renda familiar, $68,3 \%$ dos respondentes se concentram entre as classes sociais A, B e C segundo o IBOPE (2015), sendo 29,3\% de classe C (de R \$ 3.152,00 a R \$ 7.879,99), 31,7\% de classe B (de R \$ 7.880,00 a R \$ 15.759,99) e 7,3\% relativo a classe A ( $R \$ 15.760,00$ ou mais).

\subsection{Relação entre condições ambientais e percepção ambiental}

A variável percepção ambiental é influenciada por variáveis som ambiental, cores, aroma, layout, ruído e temperatura. O modelo final apresentou relações significativas com as variáveis cores, aroma, layout e temperatura. Dessa forma as hipóteses H2, H3, H4 e H7 são suportadas. Já as hipóteses H1, H5 e H6 não obtiveram suporte estatístico, conforme apresentado na tabela 1.

O modelo das relações para a variável dependente percepção ambiental positiva apresenta Odds Ratio de 2,671 para a variável cores, 1,985 para a variável aroma, 11,032 para a variável layout e 3,938 para a variável temperatura. Dessa forma, quando o consumidor percebe de maneira positiva as cores ambientes, as chances de se perceber o ambiente de forma positiva aumentam em 2,671. Já quando o aroma é percebido positivamente, as chances do consumidor perceber o ambiente positivamente aumentam 1,985, assim como as chances do consumidor perceber o ambiente positivamente aumentam 11,032 vezes caso este perceba positivamente o layout. Finalmente, caso o paciente/acompanhante perceba a temperatura ambiente desagradável, as chances de perceber o ambiente negativamente aumentam 3,938. Os valores do modelo da variável percepção ambiental estão disponíveis na tabela 1 .

Tabela 1: Coeficientes do modelo de regressão de percepção ambiental.

\begin{tabular}{ccccccc}
\hline Variáveis & B & S.E. & Wald & GL & Sig. & Exp(B) \\
\hline Cores & 0,983 & 0,393 & 6,242 & 1 & 0,012 & 2,671 \\
Aroma & 0,685 & 0,328 & 4,359 & 1 & 0,037 & 1,985 \\
Layout & 2,401 & 0,350 & 47,024 & 1 & 0,000 & 11,032 \\
Temperatura & 1,371 & 0,417 & 10,804 & 1 & 0,001 & 3,938 \\
Constante & $-3,523$ & 0,510 & 47,655 & 1 & 0,000 & 0,067 \\
\hline
\end{tabular}

Fonte: Dados da Pesquisa. 
Para avaliar a qualidade de um modelo de regressão logística, podem ser utilizados vários testes estatísticos, como a verossimilhança e os coeficientes de determinação aproximados (HAIR et al., 2009). Observa-se, todavia, que o teste que tem se mostrado mais confiável é a Estatística Hosmer e Lemeshow, no qual para um bom modelo logístico o valor encontrado para esta estatística deve exceder 0,05 (TABACHNICK; FIDELL, 2000; HOSMER; LEMESHOW, 2000).

Os índices do modelo final demonstram um bom grau de ajustamento. A estatística de Hosmer e Lemeshow ficou com valor de 0,531, acima do limite indicado por Hair et al. (2009) de 0,05 o que demonstra um resultado significativo. Os valores de ajustamento do modelo estão disponíveis na tabela 2.

Tabela 2: Índices de Ajustamento do Modelo de Regressão da Variável Percepção Ambiental Positiva.

\begin{tabular}{lr}
\hline Índices de Ajustamento do Modelo & \\
\hline Estat. Hosmer e Lemeshow & 0,531 \\
$X^{2}$ (Qui-Quadrado) & 4,132 \\
Graus de Liberdade & 5 \\
-2 Log likelihood & 261,143 \\
Cox \& Snell R Square & $39,00 \%$ \\
Nagelkerke R Square & $52,40 \%$ \\
\hline
\end{tabular}

Fonte: Dados da Pesquisa.

\subsection{Relação entre percepção ambiental e respostas emocionais}

No tocante a percepção ambiental e resposta emocional, inicialmente foi avaliada a relação entre percepção ambiental positiva e resposta emocional positiva. A hipótese $\mathbf{H 8}$ é suportada e o modelo final apresentou relações significativas com a variável resposta emocional positiva, com Odds Ratio de 3,10, conforme a tabela 3 .

Tabela 3: Coeficientes do modelo de regressão de percepção e resposta emocional positiva.

\begin{tabular}{lrrrrrr}
\hline Variáveis & B & S.E. & Wald & GL & Sig. & $\operatorname{Exp(B)}$ \\
\hline Percepção e resposta emocional positiva & 1,134 & 0,242 & 22,027 & 1 & 0,000 & 3,107 \\
Constante &,- 683 &, 173 & 15,502 & 1 & 0,000 & 0,505 \\
\hline
\end{tabular}

Fonte: Dados da Pesquisa.

Destacou-se também a mesma análise para a categoria de pacientes e acompanhantes. As relações apresentam Odds Ratio de 2,65 e 3,84 e os coeficientes do modelo citado inferior a 0,05 , conforme nas tabelas 4 e 5, confirmando a hipótese H8. Este resultado significa que, em ocasiões no qual pacientes e acompanhantes têm percepções positivas do ambiente, as chances de produzirem respostas emocionais positivas aumentam em 2,65 e 3,84 respectivamente.

Tabela 4: Coeficientes do Modelo de Regressão de percepção e resposta emocional positiva para pacientes.

\begin{tabular}{lrrrrrr}
\hline Variáveis & B & S.E. & Wald & GL & Sig. & Exp(B) \\
\hline Percepção e resposta emocional positiva & 0,974 & 0,361 & 7,297 & 1 & 0,007 & 2,650 \\
Constante & $-1,204$ & 0,269 & 20,071 & 1 & 0,000 & 0,300 \\
\hline
\end{tabular}

Fonte: Dados da Pesquisa.

Tabela 5: Coeficientes do Modelo de Regressão de percepção e resposta emocional positiva para acompanhantes.

\begin{tabular}{lrrrrrr}
\hline Variáveis & B & S.E. & Wald & GL & Sig. & Exp(B) \\
\hline Percepção e resposta emocional positiva & 1,348 &, 355 & 14,394 & 1 &, 000 & 3,849 \\
Constante &,- 198 &, 239 &, 688 & 1 &, 407 & 0,821 \\
\hline
\end{tabular}

Fonte: Dados da Pesquisa.

Em seguida foi avaliada a relação entre percepção ambiental negativa e respostas emocionais negativas. 
A hipótese H9 é suportada e o modelo final também apresentou relações significativas a variável resposta emocional negativa, com Odds Ratio de 2,60 apontado na tabela 6, ou seja, as chances do mesmo emitir respostas emocionais negativas aumentam em 2,60 vezes, indicando assim que percepção ambiental negativa geram respostas emocionais negativas pelo cliente.

Tabela 6: Coeficientes do modelo de regressão de resposta emocional negativa.

\begin{tabular}{lrrrrrr}
\hline Variáveis & B & S.E. & Wald & GL & Sig. & Exp(B) \\
\hline Resposta emocional positiva &, 957 &, 238 & 16,132 & 1 &, 000 & 2,605 \\
Constante &,- 479 &, 169 & 8,066 & 1 &, 005 & 0,620 \\
\hline
\end{tabular}

Fonte: Dados da Pesquisa.

\subsection{Relação entre estado físico e emocional, percepção ambiental e respostas emocionais}

Para analisar a relação entre estado físico e emocional, percepção ambiental e respostas emocionais, torna-se evidente a conceituação de uma variável moderadora $(\operatorname{Mod})$. Essa pode ser definida como uma variável quantitativa que afeta a direção e/ou a força da relação entre a variável independente $(\mathrm{X})$ e a variável dependente (Y), Nos modelos que utilizam variáveis moderadoras, o objetivo é verificar se uma relação entre $\mathrm{X} \rightarrow \mathrm{Y}$ é, por exemplo, reduzida a zero ou até mesmo inverter o sinal da relação $+/-$, dada a utilização de uma terceira variável Mod (BARON; KENN, 1986; PAUL; DICK, 1993). O caso proposto por Baron e Kenny (1986) de mensuração e exame dessas variáveis, utilizado nessa pesquisa, é o de uma variável independente e uma variável moderadora, ambas dicotômicas, onde é possível examinar o modelo interativo utilizando a regressão logística. Kleinbaum e Klein (2010) complementam que a interpretação dos resultados neste caso é por meio da comparação das chances (Odds Ratio). Havendo aumento nas comparações, o efeito moderador é positivo. Para contrário, o efeito é negativo. Segue abaixo Figura 2 representando o modelo teórico com dados.

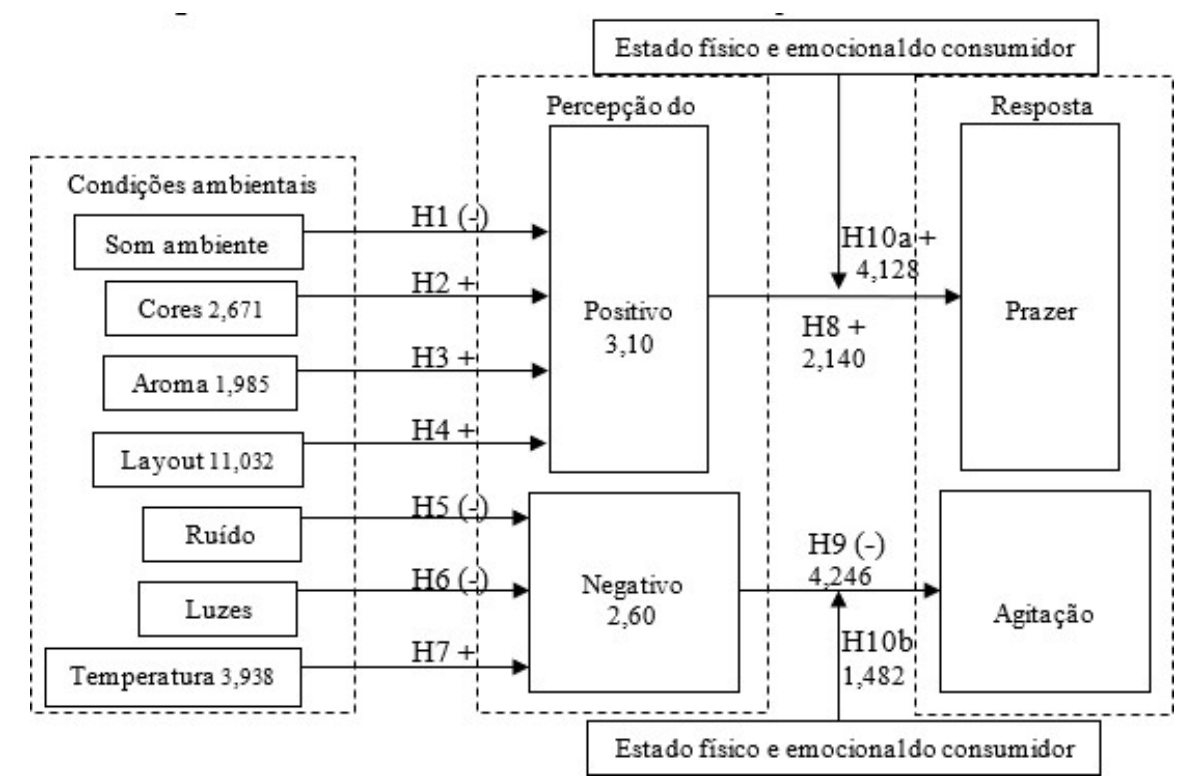

Figura 2: Modelo da influência do ambiente nas respostas emocionais do consumidor.

Fonte: Dados da Pesquisa.

Primeiramente foi avaliada a Hipótese H10a, com moderação negativa entre a variável estado físico e emocional menor que a mediana (valor igual a 0 , sendo consumidores relaxados ou sentindo pouca dor) e maior que a mediana (valor igual a 1, sendo indivíduos mais estressados ou sentindo muita dor) em a relação das variáveis da Hipótese H8, comparando a diferença entre os Odds Ratios de 4,128 e 2,140, conforme valores apresentados nas tabelas 7(1) e 7(2). Logo, a hipótese H10a é suportada. 
Tabela 7(1): Coeficientes do modelo de regressão para moderação entre estado físico e emocional positivo e hipótese $H 8$.

\begin{tabular}{lrrrrrr}
\hline Variáveis & B & S.E. & Wald & GL & Sig. & Exp(B) \\
\hline Estado físico e emocional positivo & 1,418 &, 356 & 15,867 & 1 &, 000 & 4,128 \\
Constante &,- 270 &, 247 & 1,202 & 1 &, 273 &, 763 \\
\hline
\end{tabular}

Fonte: Dados da Pesquisa.

Tabela 7(2): Coeficientes do modelo de regressão para moderação entre estado físico e emocional negativo e hipótese H8.

\begin{tabular}{lrrrrrr}
\hline Variáveis & B & S.E. & Wald & GL & Sig. & Exp(B) \\
\hline Estado físico e emocional negativo &, 761 &, 355 & 4,594 & 1 &, 032 & 2,140 \\
Constante & $-1,066$ &, 253 & 17,764 & 1 &, 000 &, 344 \\
\hline
\end{tabular}

Fonte: Dados da Pesquisa.

Em seguida foi analisado a Hipótese H10b com moderação entre o fator estado físico e emocional maior que a mediana (valor igual a 1 , sendo indivíduos mais estressados ou sentindo muita dor) menor que a mediana (valor igual a 0 , sendo indivíduos relaxados ou sentindo pouca dor) com a relação as variáveis da Hipótese H9, também comparando a diferença entre o Odds Ratio de 1,482 e 4,246, conforme valores apresentados nas tabelas $8(1)$ e $8(2)$. Portanto, a hipótese H10b é suportada.

Tabela 8(1): Coeficientes do modelo de regressão para moderação entre estado físico e emocional negativo e hipótese H9.

\begin{tabular}{lrrrrrr}
\hline Variáveis & B & S.E. & Wald & GL & Sig. & Exp(B) \\
\hline Estado físico e emocional negativo &, 393 &, 348 & 1,279 & 1 &, 258 & 1,482 \\
Constante &, 431 &, 252 & 2,924 & 1 &, 087 & 1,538 \\
\hline
\end{tabular}

Fonte: Dados da Pesquisa.

Tabela 8(2): Coeficientes do modelo de regressão para moderação entre estado físico e emocional positivo e hipótese $H 9$.

\begin{tabular}{lrrrrrr}
\hline Variáveis & B & S.E. & Wald & GL & Sig. & Exp(B) \\
\hline Estado físico e emocional positivo & 1,446 &, 366 & 15,629 & 1 &, 000 & 4,246 \\
Constante & $-1,356$ &, 272 & 24,872 & 1 &, 000 &, 258 \\
\hline
\end{tabular}

Fonte: Dados da Pesquisa.

Os resultados desse estudo revelam que há clara evidência na relação entre as condições ambientais presentes na sala de espera de uma emergência, o estado físico e emocional do consumidor e os reflexos desses na percepção e resposta emocional do consumidor. Através dos suportes apresentados nas hipóteses H2, H3, H4 e H7, torna-se relevante apontar que a sala de espera analisada apresentou em seu planejamento o uso de cores suaves, utilização de amoras artificiais e um amplo espaço entre as poltronas e móveis. No que concerne a temperatura, essa mostrou-se inferior ao ambiente em questão, gerando uma percepção ambiental negativa por parte do consumidor, evidências físicas essas que corroboram com o suporte das hipóteses apontadas. Já as hipóteses H1, H5 e H6 foram refutadas porque o ambiente da sala de espera não incluiu as condições ambientais previstas nas hipóteses: a música ambiente e o som ambiental ficou restrito ao som das televisões presentes no ambiente e o nível de ruído e iluminação apresentaram-se adequados para o ambiente analisado, questões essas que precisam ser melhores investigadas em um outro tipo de sala de espera emergencial que apresentem as condições favoráveis descritas nas hipóteses testadas. Esses resultados corroboram com a literatura apontada e evidenciam a importância do estudo e planejamento das condições físicas ambientais voltados também para uma sala de espera de uma emergência hospitalar.

\section{Conclusão}

O estudo revelou que em situações em que o consumidor tem uma percepção ambiental positiva, as chances deste responder positivamente aumentam em três vezes. O mesmo ocorreu em situações em que o consumidor percebe negativamente o ambiente, onde as chances deste responder negativamente aumentam 
em mais de duas vezes, corroborando com os estudos de Dalke et al. (2005), Solomon (2008), Zomerdijk e Voss (2010). Esses resultados trazem como uma primeira contribuição teórica um modelo teórico, conforme figura 3, que exemplifica o fato de que a percepção ambiental do consumidor deve ser levada em consideração no planejamento do ambiente de emergência hospitalar já que o consumidor é o usuário principal do serviço emergencial e sua percepção das evidências físicas ambientais estará relacionada diretamente com suas respostas emocionais a esse tipo de ambiente.

Figura 3: Modelo teórico de relações entre variáveis externas e internas no comportamento do consumidor em serviços de emergência hospitalar.

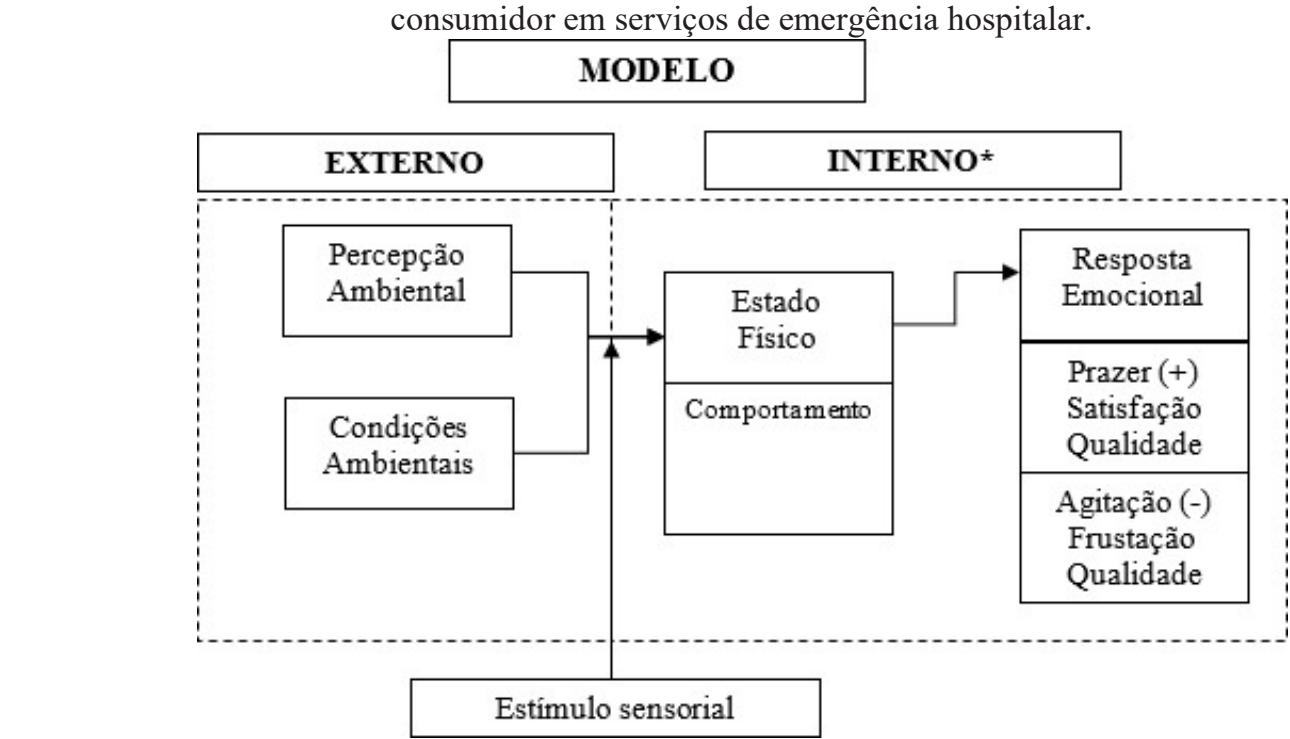

*Interno: planificação, avaliação e processamento de informação como resposta de pensamento sensorial.

Fonte: Elaborado pelos autores (2019).

Os resultados do presente estudo também apontaram, como uma segunda contribuição teórica, que o lado cognitivo racional do consumidor não é suficiente em serviços hospitalares emergenciais. A percepção da sala de espera e a resposta emocional a essa devem considerar principalmente os sentimentos dos pacientes e acompanhantes por conta de seus estados físicos e emocionais, resultado desse apoiado através dos estudos em serviços hospitalares diversos realizados por Minadeo (2010); Lee (2011), Sahoo e Mitra (2016). Através do suporte da hipótese 10a, ficou claro que em situações em que os consumidores estiverem mais estressados ou sentindo mais dor, irão perceber menos o ambiente e tenderão a produzir menos respostas emocionais positivas. Consequentemente, quando consumidores estiverem mais estressados ou sentindo mais dor, irão perceber menos no ambiente e tender a produzir respostas emocionais mais negativas, conforme suporte da hipótese $\mathrm{H} 10 \mathrm{~b}$. Assim, é provável que ao longo do tempo, as emoções negativas, que vão desde a ansiedade até a impaciência, frustração, dor e raiva consigam encobrir uma série de evidências físicas projetadas para o ambiente. Esses resultados sugerem, como terceira contribuição teórica, que um ganho significativo na eficácia do atendimento pode ser esperado se os estados físicos e emocionais dos consumidores forem sistematicamente integrados no planejamento dos ambientes de serviços emergenciais como um princípio organizador para o design e entrega de cuidados voltados para a saúde, servindo também como possíveis princípios e práticas para o gerenciamento de qualidade do serviço em questão.

Os resultados apontaram também uma quarta contribuição teórica no que concerne às percepções e respostas emocionais positivas apoiada por Beuningen (2004); Keillor, Hult e Kandemir (2004), Dalke et al. (2005), Solomon (2008) e Zomerdijk e Voss (2010). Uma vez que as emoções positivas e negativas estão fracamente relacionadas e que as estratégias para alterá-las não são a imagem espelhada umas das outras, também é importante salientar que o planejamento do ambiente de serviços pode evitar o amortecimento das emoções positivas do consumidor ao longo do tempo. Nesse contexto, a pesquisa mostrou que as percepções ambientais e respostas positivas também são influenciadas por variações das dimensões físicas de um serviço de emergência.

Uma quinta contribuição teórica pode ser observada na comparação das percepções do ambiente e respostas emocionais entre pacientes e acompanhantes, ficando claro que acompanhantes tendem a perceber 
e a responder mais positivamente ou negativamente ao ambiente do que os pacientes, já que estes apresentam estado físico e emocional mais fragilizados do que os acompanhantes. Nesse sentido, torna-se relevante abordar que tais evidências não foram encontradas em estudos anteriores.

Uma sexta contribuição teórica residiu no fato dos consumidores apresentarem alta percepção positiva do ambiente a partir do layout do mesmo, questão essa não observada em estudos anteriores relacionados aos serviços de emergência hospitalar, sendo apoiada por Sahoo e Ghosh (2016) quanto a importância do layout no conforto e promoção do bem-estar em ambientes hospitalares.

A originalidade deste estudo se baseia no fato de não existir estudos empíricos que associem os estados físicos e emocionais dos consumidores com as condições ambientes, percepção e resposta emocional ao ambiente de serviços de emergência; inexistência de estudos que realizem uma análise comparativa entre pacientes e acompanhantes em ambientes de serviços de emergência e ausência de pesquisas que reúnam, em um único estudo, as teorias relativas as condições ambientais, percepção ambiental, resposta emocional e estados físico e emocional no estudo do comportamento do consumidor em serviços de emergência hospitalar.

Do ponto de vista prático, o estudo contribui que o planejamento da atmosfera ambiental pode trazer resultados positivos em ambientes de serviços de emergência hospitalar, mas que sua inclusão na atmosfera de loja pelos gerentes requer cuidados específicos, como uma criteriosa análise da adequação das condições ambientais com os estados físico e emocional dos consumidores, destacando a importância de ambientes de emergências serem percebidos como um diferencial competitivo para as instituições de saúde já que esses são considerados áreas estratégicas por conta da captação de clientes (BOST et al., 2018).

Em termos de implicações sociais, os resultados podem ser explorados em discussões, planejamento e tomada de decisão no que concerne a parâmetros atmosféricos na indução de uma experiência afetiva mais positiva para o consumidor em um ambiente de serviços de emergência hospitalar, o que pode resultar em desempenho operacional mais eficaz do serviço.

Uma importante limitação deste estudo está em considerar, apenas como condição do ambiente, os aspectos dos sentidos da atmosfera: cor, iluminação, ruído, som, layout, temperatura e aroma. Outras variáveis da condição do ambiente, como equipamentos, material de leitura e colaboradores do hospital poderiam ser considerados como influenciadores da percepção do ambiente pelo paciente e exploradas em estudos futuros.

Uma outra limitação relevante é a abordagem transversal escolhida para condução da pesquisa. Devido às limitações de tempo, esta forma de pesquisa foi escolhida apesar de saber das suas limitações no que concerne às inferências que poderiam ser feitas a partir da condução de uma pesquisa de caráter longitudinal. Nessa linha, devido ao caráter transversal da pesquisa, foi analisada apenas a avaliação das condições ambientais pelo consumidor e não a relação entre as condições ambientais e a prestação dos serviços de emergência com a satisfação e qualidade percebida pelos clientes de forma longitudinal, relações essas que também podem ser desenvolvidas em estudos futuros.

\section{Referências}

ABREU, Fernando Araujo. Resposta emocional do paciente ao ambiente de espera em um hospital na cidade Belo Horizonte. Revista de Administração e Ciências Contábeis , Vol. 2, n. 1, 2016.

ALI, F.; KIM, W. G.; RYU, K.. The effect of physical environment on passenger delight and satisfaction: Moderating effect of national identity. Tourism Management, Vol. 57, p. 213-224, 2016.

ANAHP. Associação Nacional de Hospitais Privados. Perspectivas do setor hospitalar. Jan, 2018. Disponível em: http://anahp.com.br/sala-de-imprensa/perspectivas-do-setor-hospitalar>. Acesso em: 18 fev. 2018.

ANDERSON, Craig. Temperature and aggression: ubiquitous effects of heat on occurrence of human violence. Psychological Bulletin, Vol. 106, n. 1, p. 74-96, 1989. 
ARNEILL, Allison B.; DEVLIN, Ann S. Perceived quality of care: the influence of the waiting room environment.

Journal of Environment Psychology, Vol. 22, p. 345-360, 2002.

BABIN, J. B; ATTAWAY, J. S. Atmospheric affect as a tool for creating value and gaining share of customer. Journal of Business Research. Vol. 49, p. 91-99, 2000.

BABIN, J. B; HARDESTY, D. M.; SUTER, T. A. Color and shopping intentions: the intervening effect of price fairness and perceived affect. Journal of Business Research. Vol. 56, p. 541-551, 2003.

BACKMAN, C.; VANDERLOO, S.; FORSTER, A.J. Measuring and improving quality in university hospitals in Canada: the Collaborative for Excellence in Healthcare Quality. Health Policy, Vol. 120, n. 9, pp. 982-986, 2016.

BAILEY, Elizabeth; TIMMONS, Stephen. Noise levels in PICU: an evaluate study. Paediatric Nursing, Vol. 17, n. 10, December, 2005.

BAKER, M. J; CAMERON, M. The effects of the service environment on affect and consumer perception of waiting time: an integrative review .and research propositions. Journal of the Academy of Marketing Science. Vol. 24, n. 4, p. 338-349, 1996.

BAKER, M. J; PARASURAMAN, D. G. A. The influence of store environment on quality inferences and store image. Journal of the Academy of Marketing Science. Vol. 22, n. 4, p. 328-339, 1994.

BAKER, M. J; PARASURAMAN, A.; GREWAL, D.; VOSS, G. B. The influence of multiple store environment cues on perceived merchandise value and patronage intentions. Journal of Marketing. Vol. 66, p. 120-141, 2002.

BARON, R.M.; KENNY, D.A. The moderator-mediator variable distinction in social psychological research: conceptual, strategic, and statistical considerations. Journal of Personality and Social Psychology, Washington, Vol. 51, n. 6, p. 1173-1182, December. 1986.

BECKER, F., SWEENEY, B; PARSONS, K. Ambulatory facility design and patients' perceptions of healthcare quality. Health Environments Research and Design Journal, Vol.1, n. 4, p.35-54. 2008.

BELLIZZI, J.; CROWLEY, A. E.; HASTY, R. W. The effects of color in store design. Journal of Retailing. Vol. 59, p. 21-45, 1983.

BEUNINGEN, J.W.A. Show how you care: on the influence of the physical waiting environment on anticipated service quality. Dissertação (Mestrado) - Universidade de Twente, Enschede. 2004.

BITNER, Mary. Servicescapes: The Impact of Physical Surroundings on Customer and Employees. Journal of Marketing, Chicago, Vol.56, n. 2, p. 57-71, apr. 1992.

BOERGER, M. A. Gestão em hotelaria hospitalar. 2a ed. São Paulo: 2005.

BONE, P. F.; ELLEN, P. S. Scents in the marketplace: explaining a fraction of olfaction. Journal of Retailing. Vol. 75, p. 243-262, 1999.

BORBA, Valdir Ribeiro. Estratégias e plano de marketing para organizações de saúde. Rio de Janeiro: Cultura Médica, Guanabara Koogan, 2009.

BOST, N.; JOHNSTON, A.; BROADBENT, M.; CRILLY, J. Clinician perspectives of a mental health consumer flow strategy in an emergency department. Collegian, Vol. 25, Issue 4, p. 415-420, August, 2018.

BUJISIC, M.; BOGICEVIC, V.; YANG, W.; COBANOGLU, C.; BILGIHAN, A.; "Hobson's choice" servicescape: consumer anxiety and enjoyment. Journal of Consumer Marketing, Vol. 34, Issue: 7, 2017.

BUSTAMANTE, J. C.; RUBIO, N.; Measuring customer experience in physical retail environments. Journal of Service Management, Vol. 28, Issue: 5, 2017. 
CHAPPELL, Brian. Scent and sensibility. The Journal of Healthcare Design \& Development, Vol. 35, n. 4, p. 33-34, April, 2004.

CHEBAT. DUBÉ, L. Evolution and challenges facing retail atmospherics: the apprentice sorcerer is dying. Journal of Business Research. Vol. 49, p. 89-90, 2000.

CHEN, Chi-Shan; DING, Tsu-Jen; Consumer Satisfaction to Hospitality: A Case Study of Public Planning for Good Servicescapes in Taiwan. Journal of Tourism and Hospitality Management. Vol.3, n. 5-6, p. 113127, jun. 2015.

CHOI, Nak Hwan; JUNG, Jae Min; OYUNBILEG, Tamir; YANG, Pianpian. The impact of emotional arousal levels and valence on product evaluations: From regulatory goal perspective. European Journal of Marketing, Vol. 50, Issue: 1/2, 2016.

CHURCHILL, G. A. Jr. Marketing Research: Methodological Foundations. 7. ed. Orlando: The Dryden Press, 1999.

CORBETT, J. M. Scents of identity: organization studies and the cultural conundrum of the nose. Culture and Organization. Vol. 12, p. 221-232, 2006.

COSTA, A. L. C. N. Atmosfera de loja e o comportamento do consumidor: investigando o papel do aroma no ponto de venda. Dissertação (mestrado) - Universidade Federal de Pernambuco. CCSA, 2009.

CREMEPE. Conselho Regional de Medicina de Pernambuco. Polo médico alimenta o setor de turismo do Recife. Ago, 2014. Disponível em: <http://portal.cremepe.org.br/mostraClipping.php?id=62443>. Acesso em: 30 ago. 2015.

DALKE, Hilary et al. Colour and lighting in hospital design. Optics \& Laser Technology, 2005.

DIÁRIO DE PERNAMBUCO. Turismo médico pernambucano se intensifica com maior encontro hospitalar do Norte-Nordeste. Ago, $2014 . \quad$ Disponível em: $<$ http://www.diariodepernambuco.com.br/app/46,15/2014/08/19/ interna_turismo,523642/turismo-medicopernambucano-se-intensifica-com-maior-encontro-hospitalar-do- norte-nordeste.shtml>. Acesso em: 18 fev. 2018 .

DIÁRIO DO COMÉRCIO, 2016. Mercado de saúde cresce com as pequenas empresas. Disponível em: $<$ https://dcomercio.com.br/categoria/negocios/mercado-de-saude-cresce-com-as-pequenas-empresas $>$ Acesso em Acesso em 24 de fevereiro de 2017.

DIÁRIO DO GRANDE ABC, 2016. Setor privado de Saúde cresce no País, apesar da crise. Disponível em: <http://www.dgabc.com.br/Noticia/1967204/setor-privado-de-saude-cresce-no-pais-apesar-da-crise> Acesso em 24 de fevereiro de 2017.

DIAS, M. A. A. Hotelaria Hospitalar e sua relação com a preservação do meio ambiente. O Mundo da Saúde, ano 27, n. 4, out/dez 2003, p. 609-611.

DONOVAN, R. J.; ROSSITER, J. R. Store atmosphere: an environmental psychology approach. Journal of Retailing, New York, Vol. 58, n.1, p. 34-57, Spring. 1982.

DONOVAN, R.J. et al. Store atmosphere and purchasing behavior. Journal of Retailing, New York, Vol. 70, n. 3, p. 283-294, 1994.

EVANS, David. The effectiveness of music as an intervention for hospital patients a systematic review. Journal of Advanced Nursing, Vol. 37, n. 1, p.8-18, 2002.

ERIKSSON, H.; GREMYR, I.; BERGQUIST, B.; GARVARE, R.; FUNDIN, A.; WIKLUND, H.; WESTER, M.; SÖRQVIST, L. Exploring quality challenges and the validity of excellence models. International Journal of Operations \& Production Management, Vol. 36, Issue: 10, 2016. 
FOTLER, Myron. D. et al. Creating a healing environment: the importance of the service setting on the new customer-oriented healthcare system. Journal of Healthcare Management, Vol. 45, n. 2., p. 91-106, mar/apr. 2000.

GARCIA, Ester. Marketing da Saúde - Humanismo e lucratividade. Goiânia: AB, 2005. GIL, A. C. Métodos e técnicas de pesquisa social. São Paulo: Atlas, 1999.

GIRARD, Marc et al. The scentscape: An integrative framework describing scents in service scapes. Jbm Journal of Business Market Management, Vol. 9, n. 1, p. 597-622, 2016.

GODOI, Adalto Feliz de. Hotelaria Hospitalar e humanização no atendimento em hospitais. 2 ed. São Paulo: Ícone, 2008.

GREWAL, D. et al. The effects of wait expectations and store atmosphere evaluations on patronage in service-intensive retail stores. Journal of Retailing, Vol. 79, p. 259-268, 2003.

GROHMAN, B.; SPARENBERG, E. R.; SPROTT, D. E. The influence of tactile input on the evaluation of retail product offerings. Journal of Retailing. Vol. 83, p. 237-245, 2007.

GULDEMOND, Nick. Futuro descentralizado. Panorada ANAHP - Publicação da Anahp, Associação Nacional de Hospitais Privados, ed. novembro | dezembro, no 63, ano 12, p. 42-25, 2017.

G1, Portal. Serviços médicos do Recife atraem cada vez mais estrangeiros. Jan, 2012. Disponível em: $<$ http:// g1.globo.com/pernambuco/noticia/2012/01/servicos-medicos-do-recife-atraem-cada-vez-maisestrangeiros. html>. Acesso em: 18 fev. 2018.

HAIR Jr., J.; ANDERSON, R. E.; TATHAM, R. L.; BLACK, W. C. Análise Multivariada de Dados. $6^{\mathrm{a}}$ ed. Porto Alegre: Bookman, 2009.

HYNES, N.; MANSON, S. O som do silêncio: porque a música nos supermercados é apenas uma distração. Journal of Retailing and Consumer Services, Vol. 28, p. 171-178, 2016.

HOFFMAN, K Douglas; BATESON, John E G. Princípios de marketing de serviços: conceitos, estratégias e casos. 2a ed. São Paulo: Pioneira Thomson Learning, p. 628. 2003.

HOFFMAN, K. D.; TURLEY, L. W. Atmospherics, service encounters and consumer decision making: an integrative perspective. Journal of Marketing. p. 33-47, 2002.

HOFFMAN, K. Douglas; KELLEY, Scott W.; CHUNG, Beth C. A CIT investigation of servicescape failures and associated recovery strategies. Journal of Services Marketing, Vol.17, n. 4, p. 322-340, 2003.

HOLDER, M.; BERNDT, A.; The effect of changes in servicescape and service quality perceptions in a maternity unit. International Journal of Health Care Quality Assurance, Vol. 24, Issue: 5, 2011. 
HOSMER, D.W.; LEMESHOW, S. Applied logistic regression. 2nd edition. Wiley Series in Probability and Statistics. New York, 2000.

HOWARTH, E.; HOFFMAN, M. S. A multidimensional approach to the relationship between mood and weather. Britsh Journal of Psychology, Vol.75, p. 15-23, 1984.

HUI, M.K.; TSE, D.K. What to tell consumers in waits of different lengths: an integrative model of service evaluation. Journal of Marketing, Vol. 60, p. 81-90, 1996.

HUTTON, J., RICHARDSON, L. Healthscapes: The roles of the facility and physical environment on consumer attitudes, satisfaction, quality assessments, and behaviors. Health Care Management Review, Vol. 20, n. 2, p. 48-61, 1995.

IBGE, Instituto Brasileiro de Geografia e Estatística. Serviços. Disponível em: < http://brasilemsintese.ibge.gov. br/servicos.html>. Acessado em: 02 jul. 2016.

IBOPE, Instituto Brasileiro de Opinião Pública e Estatística. Critério Brasil inicia 2015 com nova atualização. Disponível em: <http://www.ibope.com.br/pt-br/noticias/Paginas/Criterio-Brasil-inicia-2015com-nova- atualizacao-.aspx>. Acessado em: 05 ago. 2015.

JANG, J. Y,; BAEK, E.; JUNG CHOO, H.; Managing the visual environment of a fashion store: Effects of visual complexity and order on sensation-seeking consumers. International Journal of Retail \& Distribution Management, Vol. 46, Issue: 2, 2018.

KEILLOR, Bruce D.; HULT, G. Tomas M.; KANDEMIR, Destan. A study of the service encounter in eight countries. Journal of International Marketing, Vol. 12, n. 1, p. 9-35, 2004.

KLEINBAUM, D.G., KLEIN, M. Logistic regression: statistics for biology and health. 3 rd. edition. Springler Science: New York. 2010.

KOTLER, P. Atmospherics as a Marketing Tool. Journal of Retailing, New York, v.49, n.4, p. 48-64, Winter, 1973.

LAI, K. P.; CHONG, S. C.; ISMAEL, H. B.; TONG, D. Y. K.; An explorative study of shopper-based salient e-servicescape attributes: A Means-End Chain approach. International Journal of Information Management. Vol. 34, p. 517-532, 2014.

LAM, Shun Y. The effects of store environment on shopping behaviors: a critical review. Advances in Consumer Research, Vol. 28, n. 1, p. 190-198, 2001

LEATHER P., BEALE D., SANTOS A., WATTS J., Lee L. Outcomes of environmental appraisal of different hospital waiting areas. Environment and Behavior, Vol. 35, n. 6, p. 842-869, 2003.

LEE, S. Evaluating serviceability of healthcare servicescapes: Service design perspective. International Journal of Design, Vol. 5, n.2, p. 61-71, 2011.

LEE, Andy Hee; DENIZCI GUILLET, Basak; LAW, Rob. Tourists' emotional wellness and hotel room color. Current Issues in Tourism, p. 1-7, 2016.

LOURES, Carlos A. da Silva. Um estudo sobre o uso da Evidência Física para gerar percepção de qualidade em serviços: casos de hospitais brasileiros. São Paulo, 2003. Dissertação (Mestrado em Administração) - Faculdade de Economia, Administração e Contabilidade, Universidade de São Paulo.

LOVELOCK, Christopher; WIRTZ, Jochen; HEMZO, Miguel Angelo. Marketing de Serviços: pessoas, tecnologia e estratégia. 7 ed. São Paulo: Pearson Prentince Hall, 2011.

LUNARDO, R.; ROUX, D.; CHANEY, D. The evoking power of servicescapes: Consumers' inferences of manipulative intent following service environment-driven evocations. Journal of Business Research, Vol. 69, n. 12, p. 6097-6105, 2016. 
LYU, J.; HU, L.; HUNG, K.; MAO, Z.; Assessing servicescape of cruise tourism: the perception of Chinese tourists. International Journal of Contemporary Hospitality Management, Vol. 29, Issue: 10, 2017.

MCNEILL, Lisa; MATHER, Damien. Social involvement and consumption motivation: Co-creation of magic in the servicescape. Australasian Marketing Journal (AMJ), Vol. 24, n. 4, p. 315-321, 2016

MCCOLL-KENNEDY, Janet R. et al. How do you feel today? Managing patient emotions during health care experiences to enhance well-being. Journal of Business Research, Vol. 79, p. 247-259, 2017.

MEHRABIAN, A.; RUSSELL, J. An approach to environmental psychology, MIT Press, Cambridge, MA. 1974.

MELHORES PRÁTICAS. Emergência: como resolver esse nó? Revista Melhores Práticas em saúde, qualidade e acreditação. Ano 4, ed. 16, mai/jun/jul. 2015.

MILOTIC, D. The impact of fragrance on consumer choice. Journal of Consumer Behavior. Vol. 3, n. 2, p. 179- 191(13), Dec, 2003.

MIN, D. A. et al. A rule-based servicescape design support system from the design patterns of theme parks. Advanced Engineering Informatics, Vol. 32, p. 77-91, 2017.

MINADEO, Roberto. Marketing para serviços de saúde: um guia indispensável para gestores das áreas médicas. Rio de Janeiro: Elsevier, 2010.

MOHD-RAMLY, S.; OMAR, N. A. Exploring the influence of store attributes on customer experience and customer engagement. International Journal of Retail \& Distribution Management, Vol. 45, Issue: 11, 2017.

MORRIN, M.; RATNESHWAR, S. The impact of ambient scent on evaluation, attention, and memory for familiar and unfamiliar brands. Journal of Business Research. Vol. 49, p. 157-165, 2000.

NARANG, R.; POLSA, P.; SONEYE, A. FUXIANG, W. Impact of hospital atmosphere on perceived health care outcome. International Journal of Health Care Quality Assurance, Vol. 28, Issue: 2, 2015.

NEWMAN, A. Uncovering dimensionality in the servicescape: Towards legibility. The Service Industries Journal, Vol. 27, n. 1, p. 15-28, 2007.

NILSSON, E.; BALLANTYNE, D.; Reexamining the place of servicescape in marketing: a servicedominant logic perspective. Journal of Service Management, Vol.22, n.4, p. 471-490, 2014.

NOGUEIRA, Luiz Carlos L. Gerenciando pela Qualidade Total na Saúde. Belo Horizonte: Editora de Desenvolvimento Gerencial, 2003.

OTANI, K., WATERMAN, B., FAULKNER, K., BOSLAUGH, S., \& DUNAGAN, C. (2010). How patient reactions to hospital care attributes affect the evaluation of overall quality of care, willingness to recommend, and willingness to return. Journal of Healthcare Management, Vol. 55, n. 1, p. 25-37, 2010.

PAI, P. Y.; CHARY, T. S.; Measuring patient-perceived hospital service quality: a conceptual framework. International Journal of Health Care Quality Assurance, Vol. 29, Issue: 3, 2016.

PARK, Nam-Kyu.; FARR, C. A. Retail store lighting for elderly consumers: an experimental approach. Family and Consumer Sciences Research Journal. Vol. 35, p. 316-337, 2007.

PAUL, L.S.; DICK, A. Using moderator variables in structural equation models. In: McALISTER, L.; ROTHSCHILD, M.L. (Ed.). Advances in consumer research. Provo, UT: Association for Consumer Research, Vol. 20, p. 636-640, 1930.

PETER, J. P; OLSON, J. C. Comportamento do consumidor e estratégia de marketing. 8. ed. São Paulo: McGraw-Hill, 2009. 
QUARTIER, K.; CHRISTIAANS, H.; VAN CLEEMPOEL, K. Retail design: lighting as an atmospheric tool, creating experiences which influence consumerse mood and behaviour in commercial spaces. In: Undisciplined! Design Research Society Conference. Sheffield Hallam University, Sheffield, UK, p. 1619 July 2008.

REVISTA EXAME. Uma santa guerra entre Einstein e Sírio-Libanês. Abril, 2014. Disponível em: $<$ http:// exame.abril.com.br/revista-exame/edicoes/1064/noticias/uma-santa-guerra>. Acesso em: 18 fev. 2018.

ROSENBAUM, Mark S. The symbolic servicescape: your kind is welcomed here. Journal of Consumer Behaviour, Vol. 4, n. 4, p.257-267, 2005.

SANDERS, Jeffrey L.; BRIZZOLARA, Mary S. Relatinoship between weather and mood. The Journal of General Psychology, Massachusetts, Vol. 107, p. 155-156, july, 1982.

SAHOO, Sreejesh S. Debjani; MITRA, Amarnath. Can healthcare servicescape affect customer's attitude? A study of the mediating role of image congruence and moderating role of customer's prior experience. Asia- Pacific Journal of Business Administration, Vol. 8, Issue: 2, 2016.

SAVELLI, E.; CIOPPI, M.; TOMBARI, F.; Web atmospherics as drivers of shopping centres' customer loyalty. International Journal of Retail \& Distribution Management, Vol. 45 Issue: 11, 2017.

SCHMITT, B. Experiential Marketing, Journal of Marketing Management, Vol. 15, p. 53-67, 1999.

SCHUSTER, Richard J.; WEBER, Marianne L. Noise in the ambulatory health care setting. Journal of Ambulatory Care Management, Vol. 26, n. 3, p. 243-249, july-sept., 2003.

SHARMA, A.; STAFFORD, T. F. The effect of retail atmosphere on customer's perceptions of sales people and customer persuasion: an empirical investigation. Journal of Business Research. Vol. 2, p. 183-191, 2000 .

SHENG, X.; SIGUAW, J. A.; SIMPSON, P. M.; Servicescape attributes and consumer well-being. Journal of Services Marketing, Vol. 30, Issue: 7, 2016.

SHUV-AMI, A.; SHALOM, T.; Demographic differences of perceived service quality in emergency rooms of hospital organizations. International Journal of Organizational Analysis, Vol. 25, Issue: 2, 2017.

SIDWELL, David. Living colour. The Journal for Healthcare Design \& Development. Vol. 35, n. 3, p2929, mar. 2004

SILVA, Fabiana Coêlho da; DELIBERATO, Paulo César Porto. Análise das escalas de dor: revisão da literatura.

Revista Brasileira de Ciências da Saúde. Ano VII, n. 19, jan-mar. 2009.

SOARS, B. Driving sales through shoppers' sense of sound, sight, smell and touch. International Journal of Retail \& Distribution Management. Vol. 37. n. 3, p. 286-298, 2009.

SOLOMON, Michael R. O comportamento do consumidor: comprando, possuindo sendo. 7. ed. Porto Alegre: Bookman, 2008.

SORIANO, Mirella Y.; FOXALL, Gordon R. A spanish translation of Mehrabian e Russell's emotionality scales for environmental consumer psychology. Journal of Consumer Behaviour, Vol. 2, n. 1, p. 23-36, dec. 2001.

SOUSA, Fátima Faleiros; PEREIRA, Lilian Varanda; CARDOSO, Roberta; HORTENSE, Priscilla. Escala Multidimensional de Avaliação de Dor (EMADOR). Revista Latino Americana de Enfermagem. Vol. 18, telas 01-09, jan-fev, 2010.

SOUZA, F. M. C.; SOUZA, B. C.; DA SILVA, A. S. Elementos da Pesquisa Científica em Medicina. $1^{\text {a }}$. ed. Recife: Ed. Universitária da UFPE, 2002. 
SUWARYONO, I. L.; ROSINTA, F.; SOELING, P. D.; Servicescapes Analyses of National Museum. International Journal of Administrative Science \& Organization. Vol. 20, n. 4, p. 106-112, 2013.

TABACHNICK, B.G.; FIDELL, L.S. Using Multivariate Statistics. 4th ed. Boston: Allyn and Bacon, 2001. TARABOULSI, F.A. Administração de hotelaria hospitalar. 2a ed. São Paulo: 2004.

TAYLOR, S.A.; CRONIN JUNIOR, J.J. Modeling patient satisfaction and service quality. Journal of Health Care Marketing, Vol. 14, n. 1, p. 34-44, Spring 1994.

TRIANTAFILLIDOU, A.; SIOMKOS, G.; PAPAFILIPPAKI, E. The effects of retail store characteristics on in-store leisure shopping experience. International Journal of Retail \& Distribution Management, Vol. 45, Issue: 10, 2017.

TURLEY, L.; MILLIMAN, R. E. Atmospheric effects on shopping behavior: a review of the experimental evidence. Journal of Business Research. Vol. 49, p. 193-211, 2000.

UGOLINI, M. M.; ROSSATO, C.; BACCARANI, C. A five-senses perspective to quality in hospitals. The TQM Journal, Vol. 26, Issue: 3, 2014.

VAISALA. Monitoramento hospitalar. Nov, 2015. Disponível em: < http://br.vaisala.com/br/lifescience/ applications/hospitalpharmacymonitoring/Pages/default.aspx > . Acesso em: 14 de Nov. 2015.

VALOR ECONÔMICO, 2017. Lucro das operadoras de planos de saúde sobe 70,6\% em 2016, afirma ANS. Disponível em: <http://www.valor.com.br/empresas/5001906/lucro-das-operadoras-de-planos-desaude-sobe- 706-em-2016-afirma-ans) Acesso em 24 de fevereiro de 2017.

VAN NIEKERK, B. M.; PETZER, D. J.; DE BEER, L. T. The interrelationships between boutique store atmosphere, customer satisfaction, store loyalty and repurchase intention-A Study of Females in the NorthWest Province. The Retail and Marketing Review, Vol. 12, n. 1, p. 70-88, 2016.

WAKEFIELD, K. L. et al. Retrospective: The importance of servicescapes in leisure service settings. Journal of Services Marketing, Vol. 30, n. 7, p. 686-691, 2016

WARD, James C.; BITNER, Mary J.; BARNES, John. Measuring the prototypicality and meaning of retail environments. Journal of Retailing, Vol. 68, p. 194-200, Summer, 1992.

WIRTZ, Jochen; MATTILA, Anna S.; TAN, Rachel L. P. The moderating role of target-arousal on the impact of affect on satisfaction: an examination in the context of service experiences. Journal of Retailing, New York, Vol. 76, n. 3, p. 347-365, Fall 2000.

WOLZAK, M.; Investigating the interaction between the perception of sound and color and their impact on emotions. Thesis. University Utrecht, Master Applied Cognitive Psychology. oct, 2012.

WU, T. Y.; WANG, S. G.; Effects of LED Colors Temperature and Illuminance on Costomers' Emotional States and Spatial Impressions. International Journal of Affective Engineering. v.14, n.1, p.19-29, 2015.

XIONG, J.; HE, Z.; DENG, Y.; ZHANG, M.; ZHANG, Z. Quality management practices and their effects on the performance of public hospitals. International Journal of Quality and Service Sciences, Vol. 9, Issue: 3/4, 2017.

YIN, R. K. Estudo de caso: planejamento e métodos. 3 ed. Porto Alegre: Bookman, 2005.

ZAREI, Ehsan. Service quality of hospital outpatient departments: patients' perspective. International Journal of Health Care Quality Assurance, Vol. 28, Issue: 8, 2015.

ZEMKE, D. M; SHOEMAKER, S. A sociable atmosphere: ambient scent's effect on social interaction. Cornell Hospitality Quarterly. Vol. 49, p. 317-329, 2008.

ZHU, R.; MEYERS-LEVY, J. Distinguishing between the meanings of music: when background music 
affects product perceptions. Journal of Marketing Research, Vol. 42, No. 3, pp. 333-345, Aug. 2005.

ZOMERDIJK, L. G.; VOSS, C. A. Service design for experience-centric services. Journal of Service Research. v. 13, p. 67-82, 2010. 